\title{
DYNAMIC SPACE-TIME DIFFUSION SIMULATOR IN A GIS ENVIRONMENT TO TACKLE THE COVID-19 EMERGENCY. TESTING A GEOTECHNOLOGICAL APPLICATION IN ROME
}

\author{
Cristiano PESARESI ${ }^{\text {(D) }}$, Davide PAVIA ${ }^{1}$ (D), Corrado DE VITO ${ }^{2}$ (D), Andrea BARBARA ${ }^{2,3}$, \\ Vito CERABONA ${ }^{2,3}$, Enrico DI ROSA ${ }^{3}$
}

DOI : 10.21163/GT_2021.163.07

\begin{abstract}
:
In this paper, we start from a contextualization about the measures used to contain the COVID-19 diffusion and the need to promote geotechnological proposals, data sharing and homogenous centralised systems for data collection and analysis. Successively, we present the "Dynamic SpaceTime Diffusion Simulator in a GIS Environment to Tackle the COVID-19 Emergency" that we have elaborated on the basis of the data provided by the UOC Hygiene and Public Health Service - Local Health Unit Rome 1. Particularly, after describing the main technical process able to predispose the dynamic simulator, we underline the possible added value that it can provide in terms of infection surveillance and monitoring, precision preparedness, support to decision making and territorial screening. For this demonstrative application, we have extracted from the simulator some groups of four digital screenshots which are able to show synoptic photographs in temporal perspective concerning the total number of cases of COVID-19 in Rome (Italy) for the period February 25th - September 26th. Specifically we have selected: - four screenshots for the period February 25th - June 11th, to provide significant evidence about the first three months and a half; - four screenshots for the period March 1st - March 29th, to add an insight into the geographically and statistically meaningful month of March; - four screenshots for the period June 12th September 26th, to supply an efficacious geovisualisation of the last three months and a half available; - four screenshots for the period February 25th - September 26th, to show a cumulative elaboration aimed at geolocating all the cases recorded in the seven months examined; - four screenshots for the period March 26th - September 26th, with a distinction about the first and second data sets, for a detailed (cumulative) zoom. This simulator, elaborated for the COVID-19 emergency, can be replicated in any circumstance for which specific data and information are available for the scientific community, shared and progressively updated in order to provide a productive contribution to the identification of serious infectious disease clusters, patterns and trends, and quickly respond to specific needs.
\end{abstract}

Key-words: COVID-19, Geocoding, GIS, Sanitary emergency, Dynamic simulator.

\section{INTRODUCTION}

The current pandemic of COVID-19 - declared by the WHO in March 2020 - had two epidemic waves, although a real break between the first and second waves did not occur. The WHO European region experienced its first epidemic wave in March and April 2020, which - after repeated lockdowns in many countries - seemed to be controlled during the following summer months (Kupferschmidt, 2020). The second epidemic wave started in September 2020 with a rapid escalation of cases, mainly due to government easing of restrictions and people's consequent reduction of attention to precautions (Looi, 2020).

\footnotetext{
${ }^{1}$ Department of Letters and Modern Cultures, Sapienza University of Rome, Rome, Italy cristiano.pesaresi@uniroma1.it,davide.pavia@uniromal.it

${ }^{2}$ Department of Public Health and Infectious Diseases, Sapienza University of Rome, Rome, Italy corrado.devito@uniromal.it, andrea.barbara@uniromal.it,vito.cerabona@uniromal.it

${ }^{3}$ UOC Hygiene and Public Health Service - Local Health Unit Rome 1, Rome, Italy

enrico.dirosa@aslromal.it
} 
Now we are facing the tail of the second wave, after continuous attempts to balance opening-up and lockdowns, personal freedoms and public health needs. The real turning point came with the possibility of using SARS-CoV-2 vaccines, the development of which has proceeded apace over the past year, resulting in one of the most important scientific findings and the largest mass vaccination of our century. The best comparison to the massive global vaccination effort might be the smallpox vaccination campaigns that culminated in eradicating the disease (Horton, 2021). Currently (first half of May 2021), there are 115 COVID-19 vaccine candidates; 333 are being tested in clinical trials, and 15 are approved (McGill COVID19 Vaccine Tracker Team, 2021). Globally, 8.4\% of the world population has been vaccinated with the first or single-dose and $4.1 \%$ is fully vaccinated. In Italy, of the 24,502,646 people vaccinated, 7,561,566 are fully vaccinated (Ministero della Salute, 2021).

Although the available vaccines are highly effective and safe in reducing the likelihood of serious disease, we must take several variables into account before completely abandoning primary prevention measures and resuming free movement within and between states. First of all, even though COVID-19 vaccines induce higher levels of antibodies than natural infection, scientific studies, even with the short amount of time that has passed, cannot say how long the antibodies will last. Secondly, new variants of the SARS-CoV-2 virus are sprouting up that might be more transmissible and resistant to vaccines. Finally, it is not clear whether vaccines prevent infection. If not, the achievement of herd immunity would be seriously compromised (Aschwanden, 2021). In this scenario, primary prevention measures should be maintained until vaccination coverage reaches safe levels. Most countries in the world are continuing to implement mitigation strategies, using various degrees of non-pharmaceutical interventions (NPI) combined with backward and forward tracking of people infected, and tracing their contact (TTT) interventions. Repeated lockdowns are also used as a containment strategy.

Geospatial analysis, in this context, has much room for application. Geolocalisation has proved to be an essential tool for analysing the path of the virus on a global scale and to modelling scenarios of virus distribution (Valjarević et al., 2020). In other cases, GIS technology was used to identify the space-time clusters of COVID-19 in a large country at the family level (Xu et al., 2021). Moreover, GIS, spatial statistics and specific methods have been used to study the time-series spatial autocorrelation patterns and possible factors influencing the COVID-19 diffusion at different geographical scale, in order to provide important information for the disease prevention and surveillance (Xiong et al., 2020). GIS mapping has been also used to represent and analyse some key aspects and themes, as for example (de Kadt et al., 2020): COVID-19 "Index of risk factors to maintaining social distance and preventative hygiene" and "Index of risk factors that increase health and social vulnerability during an outbreak or broader shutdown".

Such technology is most useful in the early phase of a pandemic to identify and contain outbreaks, in the middle phase to identify areas at greatest risk of spreading, and in the final phase to identify critical areas and factors. And then, during the COVID-19 pandemic, the integration among GIS elaborations, web-based tools and map-based dashboards is making it possible to: offer update accessible information; feed data sharing and knowledge; show territorial discrepancies and emergency conditions; support international comparisons and decision making; evidence the need of applicative solutions based on real-time data, also having a circumstantial level of detail (Kamel Boulos \& Geraghty, 2020; Dangermond et al., 2020).

After all, many recent researches have underlined the great analytical capabilities of specific techniques and methods in a GIS environment and in a perspective of interoperability i.e. in order to: identify possible risk factors and areas exposed to electromagnetic fields coming from radio base stations (Pesaresi \& Pavia, 2021); model and produce the acoustic map of the vehicular traffic of an urban area (Dardanelli et al., 2017); reduce emergency department (ED) overcrowding and particularly code white (inappropriate) attendances, after having identified the zones of greatest provenance of improperly used ED access (Pesaresi et al., 2020a); select and determine the suitable locations of certain elements and components (Nistor et al., 2019; Cristea \& Jocea, 2016); work towards combined approaches with machine learning and remote sensing for recognising areas strongly exposed to hazard and risk (Avand et al., 2021) and coming up with a cognitive system in response to emergencies (Atek et al., 2021). 
In a similar framework and in the light of the possible added value obtainable by geotechnological applications, a dynamic space-time diffusion simulator in a GIS environment, based on official health data regarding Rome (Italy), is presented in this study.

\section{THE IMPORTANCE OF GEOTECHNOLOGICAL PROPOSALS, DATA SHARING AND HOMOGENOUS CENTRALISED SYSTEMS}

In a previous piece of work the importance has been evidenced of having a geotechnological tested system that (in an adequate balance with privacy aspects) can make it possible to produce detailed digital maps, applications and models able to support geospatial analysis, bringing to light crucial aspects, trends, patterns and clusters. In particular, three geotechnological proposals (Fig. 1) were hypothesised to tackle health emergencies starting from the present COVID-19 pandemic, with specific reference to the (Pesaresi et al., 2020b): elaboration in a GIS environment of geographicalhealthcare-epidemiological models of spatial and temporal diffusion; devising of an App for data tracking, digital flow mapping and health education; realization of a geolocalised online questionnaire, for a smart survey for the identification of possible positives. One of the biggest and most common problems for the scientific community has been the lack of highly detailed accessible and continuously updated data for testing and advancing geotechnological solutions able to support interpretative analysis, intervention planning, definition of implementational measures.

The low level of data disaggregation and the absence of an open-source platform for collecting and sharing accurate data in real-time - although we are (or should be) in the era of big data - do not make it possible to scientifically operate and contribute to the decision making and strategic planning of sanitary actions. Therefore, the potentialities of applied geography and the GIS approach, in the interdisciplinary perspective, risk being suffocated by the absence of adequate data, with notable negative repercussions on the geospatial analysis and operative steps. In a similar situation, three geotechnological proposals were advanced on the basis of simulated data and theoreticalmethodological assumptions aimed at showing the possible added value and what could be produced at application level to support emergency phases in the case of availability and sharing of real data.

Specific examples and demonstrative elaborations were presented, for conventionally selected study areas of Rome (Italy), in order to underline the potential contributions that could be provided as support to intersectoral analysis, policy making and precision preparedness.

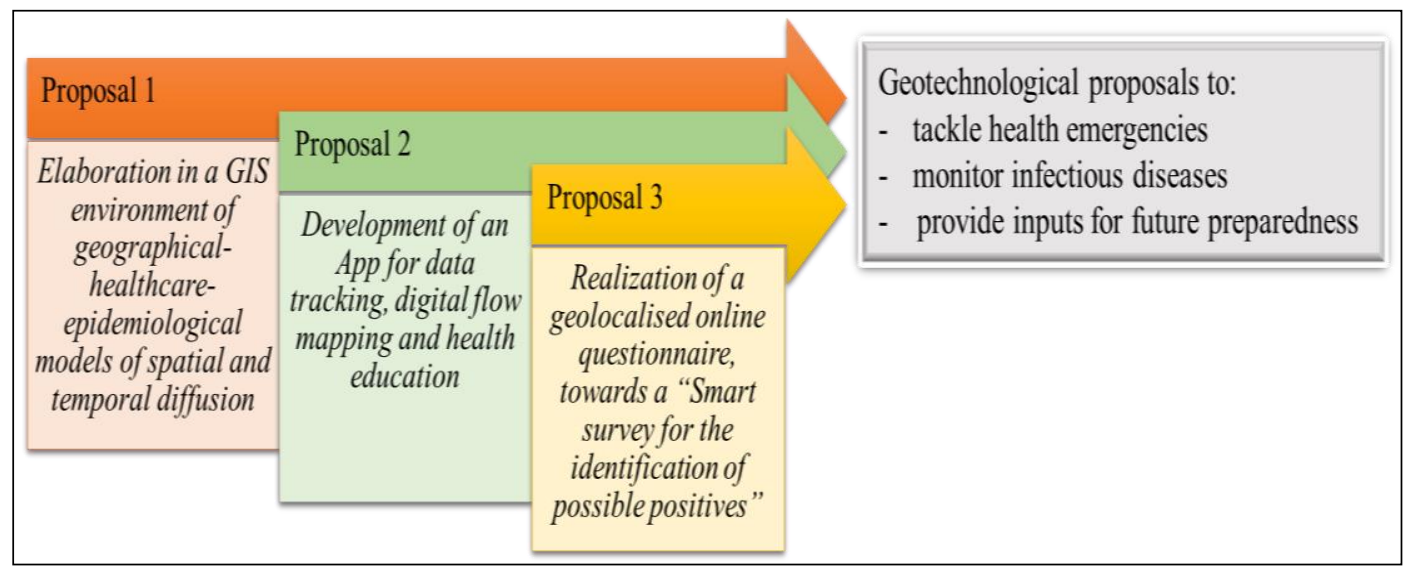

Fig. 1. Three geotechnological proposals to tackle health emergencies, monitor infectious diseases, provide inputs for future preparedness starting from the COVID-19 pandemic. Source: Authors' elaboration based on Pesaresi et al., 2020b. 
After all, other studies have generally reiterated that:

- reliable disaggregated and age-disaggregated data - above all in the case of COVID-19 - are vital for epidemiological and geographical analysis, supporting specific responses and monitoring the prioritisation of different age groups in policy actions (Lloyd-Sherlock et al., 2021, 141);

- a centralised, harmonised and fully reproducible open-access and age-structured database of COVID-19 is essential to estimate the impact of the disease, allow comparisons and support scientific analysis which can provide a notable added value also for practical measures and future preparedness (Riffe et al., 2020, 1-2);

- real-time and valid information flow and reporting have a crucial role during pandemics and principles of open science and data sharing are of utmost relevance (Wolkewitz \& Puljak, 2020, 2-3);

- a common insight that an invaluable benefit could come from a pooled, accurate and available dataset analysable by the scientific community in academic and research institutes is necessary (Cosgriff et al., 2020);

- it is hoped that open science will start to be considered as a "new normal" scientific method in this and future crises too, since it can greatly help to find approaches and strategies to alleviate global and individual suffering (Tse et al, 2020, 5);

- geography, GIS and an interdisciplinary approach can play a central role in monitoring and mitigating disease transmission, above all if it is possible to refer to a plethora of tested methods and functionalities which can be implemented and refined in a short timeframe to effectively interact with public health institutions, decision-makers and local officials "about spatial and space-time transmission dynamics" (Desjardins et al., 2020, 6);

- incorporating and integrating geographic information science and technology (GIS\&T) into COVID-19 pandemic surveillance and modelling helps to understand and monitor the disease. Particularly, applications and elaborations "of GIS\&T include 1) developing spatial data infrastructures for surveillance and data sharing, 2) incorporating mobility data in infectious disease forecasting, 3) using geospatial technologies for digital contact tracing, 4) integrating geographic data in COVID-19 modeling, 5) investigating geographic social vulnerabilities and health disparities, and 6) communicating the status of the disease or status of facilities for return-to-normal operations" (Smith \& Mennis, 2020, 1).

In another contribution (Pesaresi, 2020), the prompt availability and setup of automatically updated data regarding COVID-19 - and for the future about other possible infectious diseases - have been considered as a key element in order to support accurate GIS mapping and interdisciplinary analyses able to allow the interpretation of viral and dynamic phenomena and to validate the response capacity of the healthcare facilities, hospitals, emergency departments, intensive therapies and governments. In this perspective, a common and determined effort should be necessary for the creation of a tested Homogenous Centralised System for Dynamic Geoprocessing and Geolocalised Diffusion Models in response to Emergencies (Fig. 2) into which to collect and merge data (involving domicile and residence of the positives, their age and sex) gathered by means of homogenous formats in order to set up reliable and superimposable databases at a regional, provincial and municipal level, such as for census sections. Emergency departments, Local Health Units and the other health facilities should in fact have recourse to a standard system and process of data collection that flows into a highperformance cloud platform, able to support geocoding and geospatial analysis, reducing time and risk of error, guaranteeing requirements of safety and confidentiality. Through geolocalised data, which make it possible to use the geocoding tool in GIS environment on the basis of domicile and residence, specific models of spatial and temporal diffusion can be elaborated and updated to recognise - on satellite images, digital street maps or other bases - critical areas and axes, also by the overlay of multiple layers. Moreover, in terms of synthetic view and to avoid confidentiality matters, from the point maps, where each point can for example represent the single ascertained case or death, it is possible to move towards aggregate scale digital mappings or geospatial and geostatistical functionalities, to identify the zones requiring ad hoc interventions and services. 


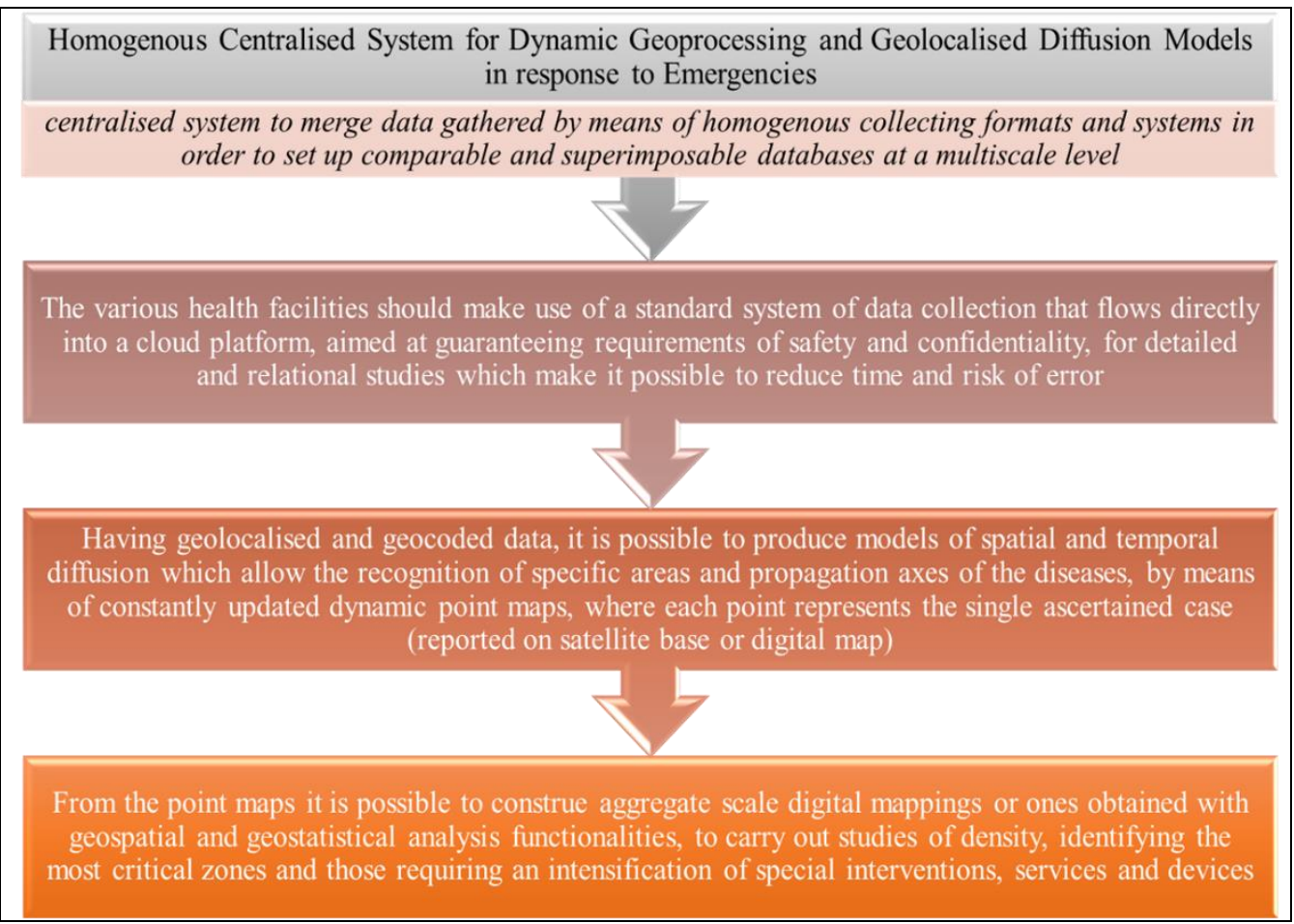

Fig. 2. The importance of having a Homogenous Centralised System for Dynamic Geoprocessing and Geolocalised Diffusion Models in response to Emergencies. Source: Authors' elaboration based on Pesaresi, 2020.

\section{MATERIAL AND METHODS. DYNAMIC SPACE-TIME DIFFUSION SIMULATOR IN A GIS ENVIRONMENT TO TACKLE THE COVID-19 EMERGENCY}

\subsection{Prerequisites and basic aspects}

In the group of interdisciplinary operative works aimed at highlighting how the GIS applications and digital models can prove to be of great support for research and concrete actions aimed at opposing contingent sanitary problems and social drama, through rigorous processing and analysis of data and images, we have produced and tested a "Dynamic Space-Time Diffusion Simulator in a GIS Environment to Tackle the COVID-19 Emergency" on the basis of the data collected and provided by the UOC Hygiene and Public Health Service - Local Health Unit Rome 1 (UOC Servizio Igiene e Sanità Pubblica - ASL Roma 1). This simulator, created specifically for the COVID-19 emergency, can be replicated in any circumstance for which accurate data and information are available, shared and progressively updated and it has been conceived to provide a significant contribution to recognise outbreaks of infectious diseases, identify clusters and distribution patterns, monitor and predict the disease's temporal and spatial evolution.

As a first foundation, the simulator, developed in a GIS environment on satellite images, digital street maps and other background templates, is based on the geocoding process, by means of which data and information, with a precise domicile and residence address available, are geolocated to show the evolution of the phenomenon, the spatial distribution of positive cases and deaths and to conduct a high-detail screening. It (potentially) makes it possible to daily monitor the progression of the disease, in terms of territorial thickening and temporal trend, through automatic and digital GISmapping able to auto-implement in parallel with the evolution of the phenomenon itself and with the registration of the data in a specific database. 
The dynamic simulator is also characterised by an animation effect, to be interpreted like a video mode, which can make it possible to select and represent the data and their continuous evolution for specific temporal ranges, supporting advanced hypotheses on possible disease evolution and revealing, in terms of retrospective analysis useful as training for the future, which measures and interventions could have recorded with a certain level of success to tackle the sanitary emergency being studied. The role played by the simulator can be notable in different phases but above all in the early stages of an emergency, to concretely work and take decisions in order to rapidly block the spread of the disease through specific analysis, guidelines and ad hoc measures with which to intervene on the first affected areas and the ones highly exposed to contagion risk.

\subsection{The source and characteristics of the data}

The Public Health and Hygiene Service (SISP) is one of the Services of the Prevention Departments of the Local Health Authorities (ASL). Starting from February 2020, the SISP of ASL Rome 1 was involved in the management of the SARS-CoV-2 pandemic and set up a work team that could guarantee the tracing of positive patients permanently resident in the ASL Rome 1 territory or even temporarily present and admitted to hospitals in the area of competence.

From a procedural point of view, the multidisciplinary team activated a management system with the use of an e-mail box (Covid19@aslroma1.it) appointed to receive, from the hospitals and general practitioners of the area and from other Hygiene Services, reports of positivity to COVID-19 through the form ANNEX_3_SCHEDA_SARS_COV2_v4.1, specially modified and adapted by SERESMI for the notification of the infectious disease from SARS-CoV-2. Every day an administrative resource had the task of monitoring the e-mail address Covid19@aslroma1.it and to print the reporting forms.

For the registration of the notification forms a database in blocks was created, always accessible to all UOC SISP's staff (Doctors, Health Assistants, Environmental and Workplaces Prevention Technicians and Nurses) for the daily care of the domiciled patients and for Contact Tracing operations, which contains the following information.

- The first block contains the patient's personal information and other information useful for optimising the management of the case and specifically: progressive master is the progressive arrival number of the ANNEX_3_SCHEDA_SARS_COV2_v4.1 email address at Covid19@aslroma1.it; gender; place and date of birth; the date reported on the ANNEX_3_SHEET_SARS_COV2_v4.1 form which corresponds with the positive result of swab test carried out; date of input of the information on the database; Official Body that notified the infectious disease; indication of the place of hospitalisation or home isolation, and home address; Municipality and Local Health Authority competent for the patient's residence. Patient Tax Code, surname, first name, telephone number and e-mail address of the patients, useful for the daily activation of health surveillance and for contact tracing activities, as well as for official communications between the Local Health Authority and the patient, were removed from the dataset used for the analysis to ensure anonymity.

- The second block contains information on the evolution of the patient's clinical picture after a second control swab, and specifically: ESITO_1 which contains the swab result or the clinical evolution of the patient (Healing, Clinical Healing, Discharge, Death); Note_Esito_1 which contains information on the outcome date (date of Healing, date of Discharge, date of Death), Data Segnalazione Aggiornamento_1 which indicates the Date of notification of the update indicated on the notification form (corresponding to the second swab carried out or with the exitus date or with the discharge date from the Hospital), the data input date Aggiornamento_1, which is the date on which such notification reached the Hygiene Service and was taken charge of; the Notifying Body_1 and the new placement of the Patient through the information contained in the "Isolation / Hospitalization at_1" cell.

The subsequent blocks represent further evolutions of the clinical status or consequent to following controls with TNF. 


\subsection{The data cleaning and optimisation}

In order to convert the COVID-19 cases into an accurate feature class - a vector spatial data format used to represent and analyse a discrete object in a GIS environment - a data cleaning and optimisation (aimed at geocoding) was at first conducted on the data sets received from the Local Health Unit Rome 1.

Basically, the operation was conducted with regard to two criteria: a temporal one, to verify the presence of the notification dates for each of the patients recorded; a spatial one, to verify the presence and the quality of the addresses of the place where patients spent their isolation. Both pieces of information were required for the creation of a simulator aimed not only at representing the location of the cases, but also their change over time, bringing together the dimensions of space and time in a 4D application devised to monitor and study the spread of the outbreak.

Since the data were provided in two different sets with the same schema (from February 25th to June 11th 2020; from June 12th to September 26th 2020), a new Excel worksheet was created to merge the relative rows in a new database, which finally contained 3,717 rows.

Then, the data cleaning and optimisation started from the temporal criteria. The date of each notification was stored in a homonym field called "Date of notification", reporting the year, the month and the day when the case was ascertained. At first, the cells of this field were filtered to detect the presence of null values, like empty cells or values in a non-date format. Thus, the operation led to the replacement of the values of 40 cells with those of the cells of the "Date of insertion" field, which stored the date when the notification was recorded into the database. Only one row was cancelled as there was no valid information in both fields, reducing the number of rows to 3,716.

These rows were then cleaned and optimised with regard to the spatial criteria. Among the spatial fields stored in the table, such as the one containing the patients' country of birth, the "Domicile" field was used as input for the geocoding process, because it contained the larger scale information available for the geocoding: the address of the places where the patients spent their isolation.

Therefore, the process has focused on reviewing both the presence and quality of these values, in order to provide the best possible input for the ESRI "ArcGIS World Geocoding Service", a cloudbased service that converts the addresses of 153 world countries into point features ${ }^{4}$. At first, the domicile field was filtered for empty cells to be deleted, reducing the number of rows to 3,713. The remaining cells were then filtered again with a specific formula, aimed at displaying only the addresses that did not start with the words "Via" and "Piazza" (i.e. the Italian words for street and square). Thus, the data cleaning and optimisation could have been concentrated on a smaller set of cells ( 297 cells, the $8 \%$ of the whole dataset), whose values were most likely not an address or a value that could be geocoded. Basically, these values were wrong for the following reasons: 31 cells reported the country of origin of the patients instead of their address of domicile; 4 cells were filled with the value "Not specified"; 7 cells mentioned the state of the person, such as homeless, soldier and tourist, without reporting any address to geocode.

Some of the remaining filtered cells reported more than one address and were rewritten by choosing the first: this has generally been the case of the cells of patients treated by more than one medical facility. The remaining 3,671 rows were cleaned from those containing addresses located outside Rome using the values of the "Municipality" field, which stored both the number of submunicipal areas in the case of Rome addresses and the name of the municipality for the others. The numbers of the sub-municipal areas were then replaced with the word "Rome", in order to refer the addresses of the "Domicile" field to the municipality to which they belong. Finally, the "Domicile" field was reviewed in search of special characters like brackets, often used to add a note to the case, in order to leave in the cells nothing but the addresses.

\footnotetext{
${ }^{4} 153$ countries are classified by ESRI "ArcGIS World Geocoding Service" from level 1 to 3, where level 1 means maximum accuracy for the geocoding service. The remaining countries of the world are classified with level 4, which means that the geocoding service is only available for points of interest and administrative boundaries. The service documentation is available at https://doc.arcgis.com/it/arcgis-online/reference/geocodecoverage.htm; last access on April 26th 2021.
} 


\subsection{The input data for the dynamic space-time diffusion simulator}

At the end of the data cleaning and optimisation, the 3,184 remaining rows were copied onto a new database with a more simplified schema than the one of the original source, made up only of the fields that would be used by the simulator: the "Date of notification", the "Domicile" and the "Municipality" ones. The two latter fields where then used as the input ones for the "Geocode Addresses" tool of ArcGIS Pro, the latest desktop application of the ArcGIS platform. Using ESRI's "ArcGIS World Geocoding Service" as the input address locator, the "Domicile" field was referred as the Address or Place parameter of the tool, while the "Municipality" field was used as the City one. Finally, "Italy" was chosen in the Country parameter to reduce the extent of the geocode process.

Each of the points created by the geocode process was evaluated in terms of the accuracy of the geocoding: of all the addresses, $6.5 \%$ were rated as "Unmatched", which means the process could not find a perfect match for these input addresses. In most cases, these addresses did not match due to the lack of the civic number or the use of a different prefix than the one found by the address locator (i.e. "Street" instead of "Boulevard"); furthermore, they did not match because of the presence of homonymous addresses. Each of these 207 addresses was rematched every time it was possible to find an unambiguous candidate among the ones selected by the process. $\mathrm{T}$

The point feature class was then added to a new ArcGIS Pro map, together with the layer of Rome sub-municipal areas downloaded from the portal of the Italian National Institute of Statistics (ISTAT) $)^{5}$, in order to select and delete the points outside the city boundaries using the "Select Layer by Location" tool, reducing the number of features to 3,056. These were the points that were used as input for the time-space simulator, which used the values of the "Date of notification" field to gradually display the points based on a time slider. This feature can be applied to all temporal layers of a map, that is to say the ones that point to a dataset that stores time values in its attribute table. Using these values, the COVID-19 case layer could be turned into a temporal one and could be used to display the gradual increase of the cases via the time slider.

Using for example a frequency of two days, an animation was created based on the values of the "Date of notification" field, showing the spread of the cases in Rome from February 25th to September 26th. Due to the possibility to replay the animation with a different time interval, this tool can be particularly useful to support the study of the outbreak and its spread, slowing down the frame rate of the animation to better identify the distribution of the clusters.

Finally, the animation was exported as an independent video file, in order to reach a wider audience and effectively communicate the spread of the infection across the space and time studied.

\subsection{The temporal scanning for the demonstrative application}

The data used for producing and testing the simulator, originally received in two sets, cover two successive periods and make it possible to analyse the dynamics recorded in about seven months.

- From February 25th to June 11th 2020 (in terms of notification date) and involving the period of the Italian lockdown; in fact, on the basis of the DPCM dated 9th March 2020, it was declared that the urgent measures to contain the contagion would have been extended throughout the whole national territory (Official Gazette General Series No. 62; https://www.gazzettaufficiale.it/eli/id/2020/03/09/20A01558/sg), and the lockdown period continued until May 18th, the day of the end of the enforcement of all restrictive measures on circulation within the regional territory on the basis of Decree-Law No. 33 dated 16th May 2020 , (https://www.normattiva.it/uri-res/N2Ls?urn:nir:stato:decreto.legge:2020-05$16 ; 33$ !vig=).

\footnotetext{
${ }^{5}$ https://www.istat.it/storage/cartografia/basi_territoriali/aree-subcomunali-xls-shp/ASC2011_WGS84.zip; last access on May 1st 2021.
} 
- From June 12th to September 26th 2020 and involving the period of the second wave inflated during the summer holidays, the recovery of touristic flows sometimes without respect for the rules and the easing off of the containment measures.

For the demonstrative application shown here we have focussed the attention on the cases of positivity for each period and the cumulative cases, representing the number of positives ascertained in one of the healthcare facilities of Local Health Unit Rome 1.

Particularly:

- for the period from February 25th to June 11th 2020, we have used orange points to represent each positive ascertained, using a satellite image as background template;

- $\quad$ for the period from June 12th to September 26th 2020, we have used red points to represent each positive ascertained, using a digital street map as background template;

- $\quad$ for the period from February 25th to September 26th 2020, we have used overlayed orange and red points to show each positive ascertained, using Light Gray Canvas as background template in order to better highlight the notable number of cumulative points which also have two different colours. The first screenshot, dated June 12th, is here due to the sum of the cases recorded from February 25th to June 11th plus the cases recorded on June 12th.

\section{RESULTS AND DISCUSSIONS}

\subsection{The digital screenshots extracted by the simulator}

For the demonstrative application and analysis we extracted by the simulator - displayable and to be seen online in a simplified and exemplificative mode at http://geolab.uniroma1.it/geolab/it/simulatorecovid19 - some groups of four digital screenshots which are able to provide synoptic photographs in temporal perspective regarding the number of cases of COVID-19, on the basis of the data available and subject to data cleaning and optimisation and then to geocoding.

Specifically, we have selected the following.

- Four screenshots for the period February 25th - June 11th (Fig. 3a), represented over a satellite image (and with sub-municipal areas of Rome), to provide incisive evidence about the first three months and a half available with the cadence: February 25th, April 11th, May 11 th, June 11th.

- Four screenshots for the period March 1st - March 29th (Fig. 3b), over a satellite image, to give an insight into the month of March (geographically and statistically very meaningful and not graphically covered in the previous elaboration) with the cadence of March 1st, March 8th, March 22nd, March 29th.

- Four screenshots for the period June 12th - September 26th (Fig. 4), over a digital street map, to provide an efficacious geovisualisation of the last three months and a half available with the cadence: June 12th, July 26th, August 26th, September 26th.

- Four screenshots for the period February 25th - September 26th (Fig. 5a), over a Light Gray Canvas as background template, to produce a cumulative elaboration aimed to show all the cases recorded in the seven months examined, with a distinction regarding the first and the second data sets and with the cadence: June 12th (starting from February 25th), July 26th, August 26th, September 26th.

- Four screenshots for the period March 26th - September 26th (Fig. 5b), over a Light Gray Canvas, with a focus on a zone of Rome centre, with a distinction regarding the first and the second data sets, to evidence at a detailed geographical scale the potentialities of a similar application in terms of accurate territorial screening and surveillance, on the basis of the bimonthly cadence March 26th, May 26th, July 26th, September 26th. 

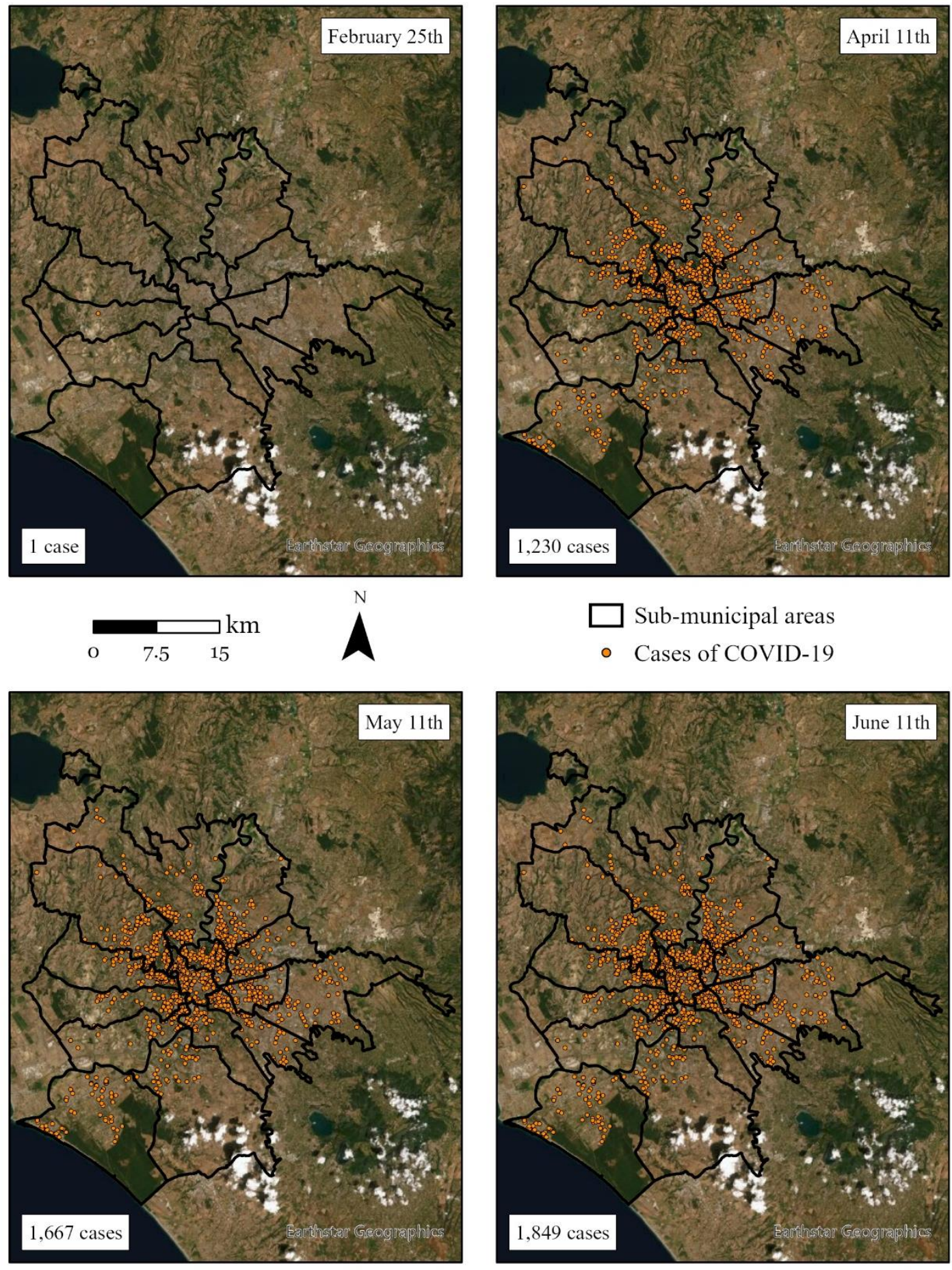

Fig. 3a. Four screenshots extracted by the dynamic space-time diffusion simulator in a GIS environment regarding the number of cases of COVID-19 in Rome, for the period February 25th - June 11th, on the basis of the data provided by the UOC Hygiene and Public Health Service - Local Health Unit Rome 1, over a satellite image and with sub-municipal areas of Rome.

Source: Authors' elaboration. 


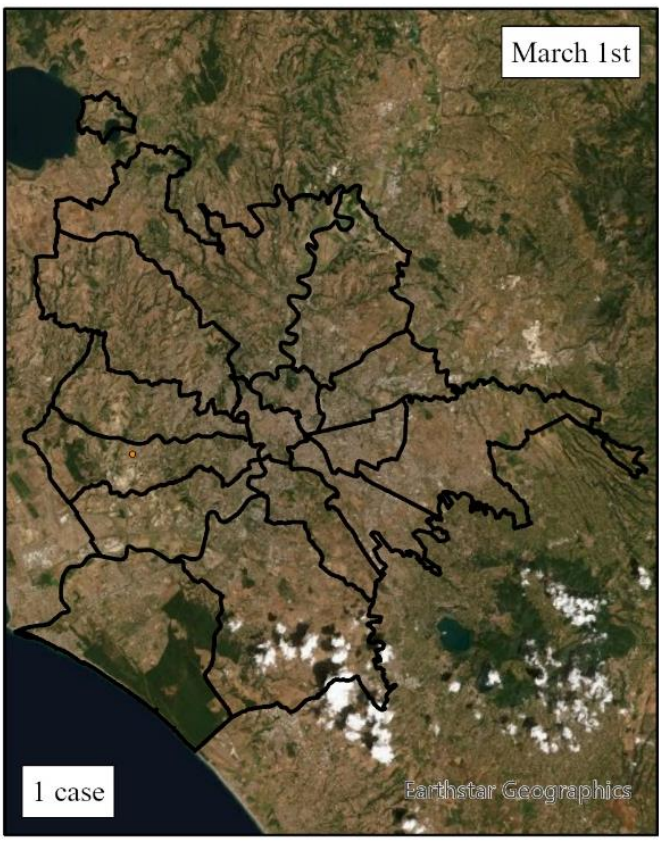

$\mathrm{N}$

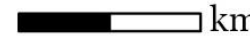

o $\quad 7.5 \quad 15$

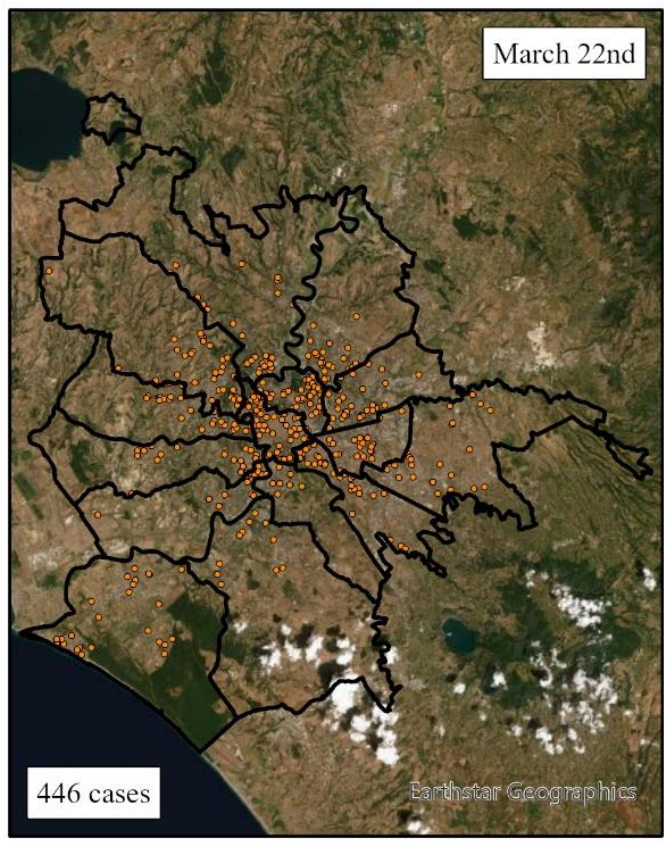

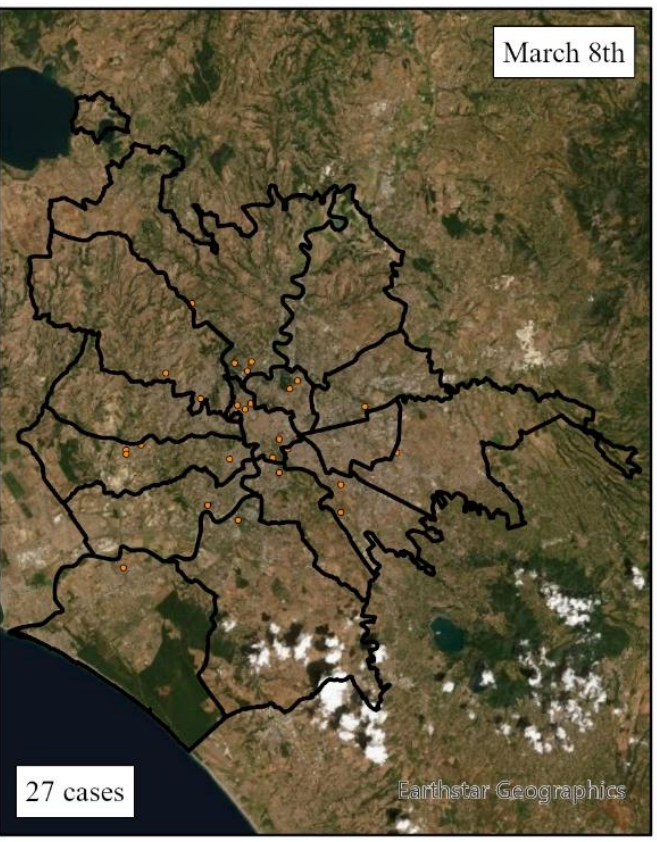

Sub-municipal areas

- Cases of COVID-19

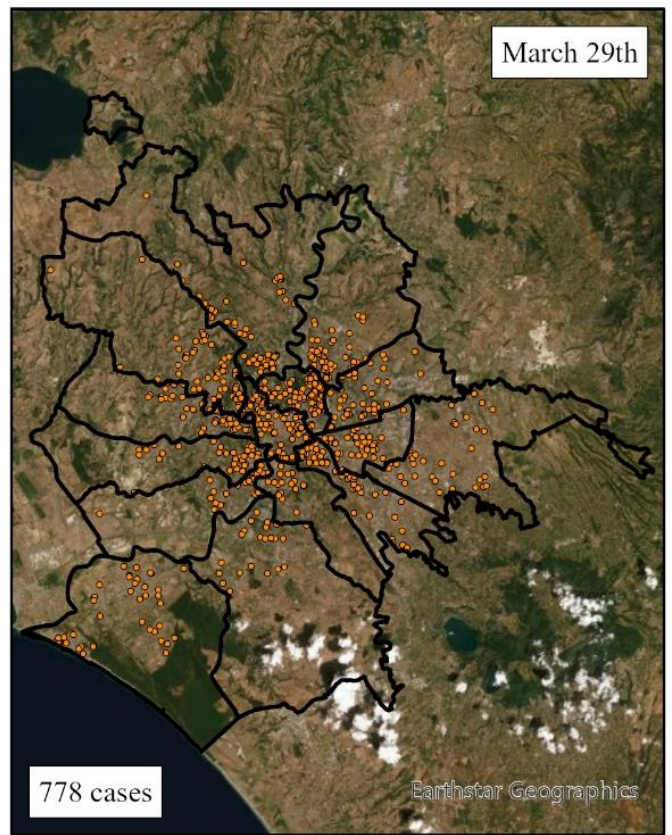

Fig. 3b. Four screenshots extracted by the simulator regarding the number of cases of COVID-19 in Rome, for the period March 1st - March 29th, on the basis of the data provided by the UOC Hygiene and Public Health Service - Local Health Unit Rome 1, over a satellite image and with sub-municipal areas of Rome. Source: Authors' elaboration. 


\subsection{Discussions and results}

\subsubsection{The period February 25th - June 11th, and a focus on March}

Fig. 3a first of all shows the passage from the first COVID-19 case recorded in Rome on the basis of the data available, in a sub-municipal area in the west sector, to the 1,230 cases recorded on April 11th, that it is to say about one month and a half later. In this period the diffusion of COVID19 was numerically greatly accelerated and spatially spread out, with a high concentration in the centre but with several clusters, thickening and axes in different sub-municipal areas. Fig. 3a moreover shows the subsequent slowing down which led to 1,667 cases on May 11th, while according to the previous trend a notably greater number of cases could have been recorded. Therefore, it is worthy to note the positive effects determined by the lockdown which make it possible to obstruct a widespread diffusion that could have been even more dramatic.

The positive effects of the lockdown and other measures (social distancing, devices like face masks etc.) were particularly remarkable in the successive months, since on June 11th 1,849 cases were recorded, with a contained increase of the cases.

Fig. 3b - which can be considered a geographical lens on the month of March, in order to understand the dynamic in this delicate period - shows the still small number of cases (27) on March 8th and makes it possible to have a detailed picture of their geolocalisation, clearly evidencing the potential added value of having similar applications in real-time to support decision making, to arrange the interventions into a hierarchy, to deploy specific facilities and sanitary services around the territory. Fig. 3b also shows that in the successive weeks the increase of the cases was rapid (446 and 778, respectively on March 22nd and 29th) also because the effects of the lockdown and ad hoc measures had a temporal phase shift and in the proximity of the cases already recorded on March 8th a series of new cases were added.

\subsubsection{The period June 12th - September 26th}

Fig. 4 first of all shows the sharp rise in the COVID-19 diffusion during the summer months. Specifically, in terms of evolutionary dynamic, it is possible to see the geolocalisation of 7 cases on June 12th and then the 172 cases on July 26th, the 544 cases on August 26th and the 1,207 cases on September 26th. As far as concerns the spatial distribution, the cases were mainly condensed into the Great Ring Road (Grande Raccordo Anulare), with a particular thickening in the central-northwestern sector since August 26th. Fig. 4 also puts in evidence the fact that, in this period, there was a slow increase in the first month and a half, owing to the last effects of the previous months and measures and a certain psychological fear, while the increases became faster and faster in August and September, also laying the potential bases for a rapid diffusion in the successive months. The concomitance between the easing of the restrictive measures and the progressive psychological recovery, and the connected desire to return to normality, relights a fuse in August and September that had previously been kept under control and able to break out into an accelerated rhythm.

\subsubsection{The period February 25th - September 26th, and a focus with bimonthly cadence on a zone of Rome centre}

Fig. 5a shows the combination of the data sets, in a cumulative way, through an overlay layer and makes it possible to follow the passage from 1,856 cases on June 12th (1,849 until June 11th and 7 on June 12th), to 2,021 cases on July 26th (1,849 until June 11th and 172), to 2,393 on August 26th (1,849 until June 11th and 544), to 3,056 on September 26th (1,849 until June 11th and 1,207). Therefore, the screenshot concerning June 12th represents the trait d'union among the two available data sets, the screenshot regarding July 26th shows a summation of orange and red points which slowly advance, while the screenshots concerning August 26th and September 26th put in evidence previous and new diffusion areas and axes, in a general mixed framework, with a notable overlapping of cases. 


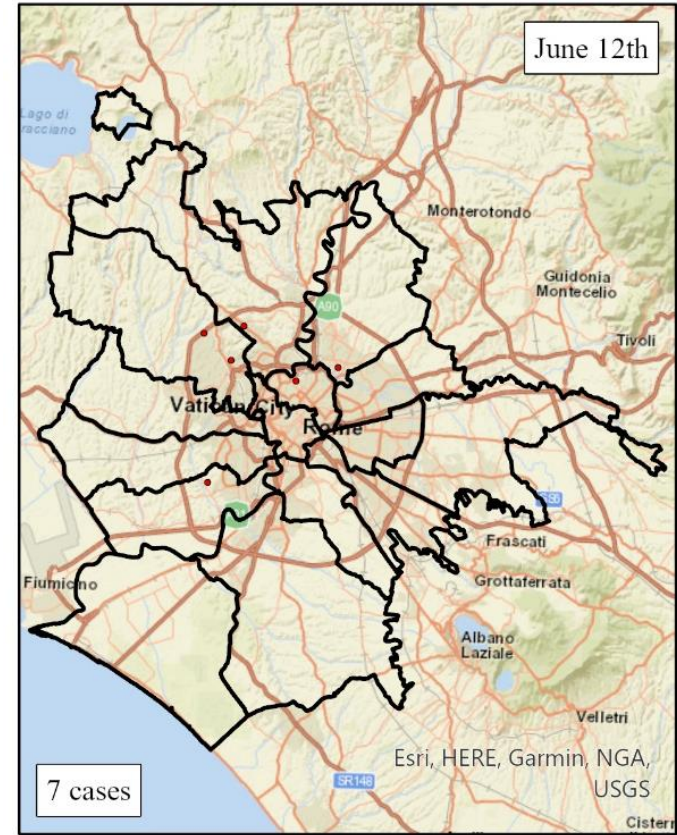

$\hat{\Lambda}$

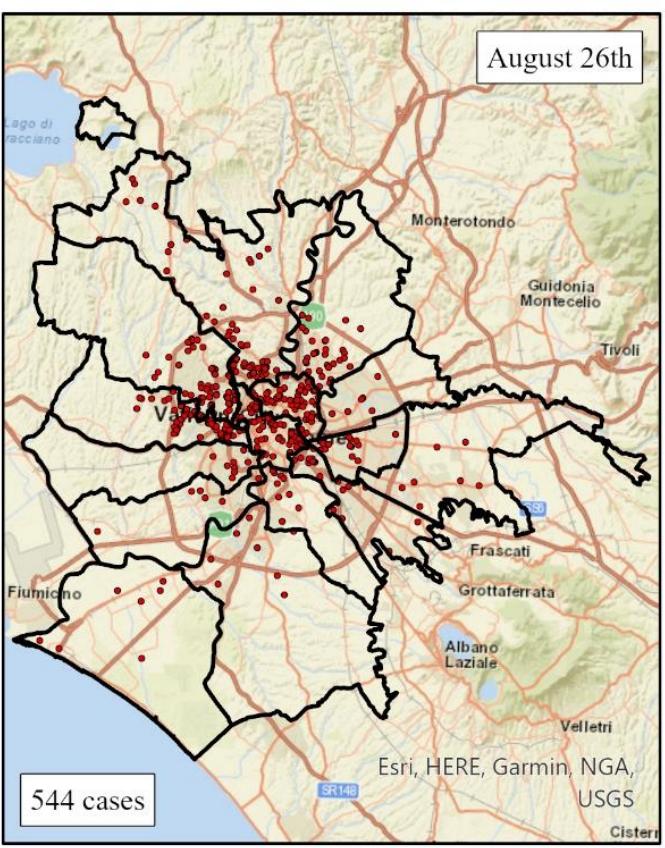

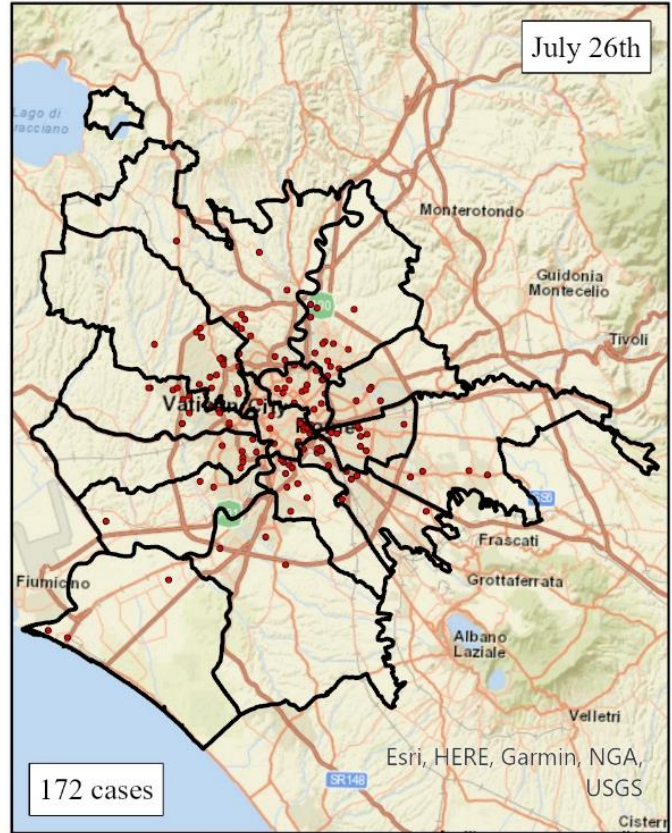

Sub-municipal areas

- Cases of COVID-19

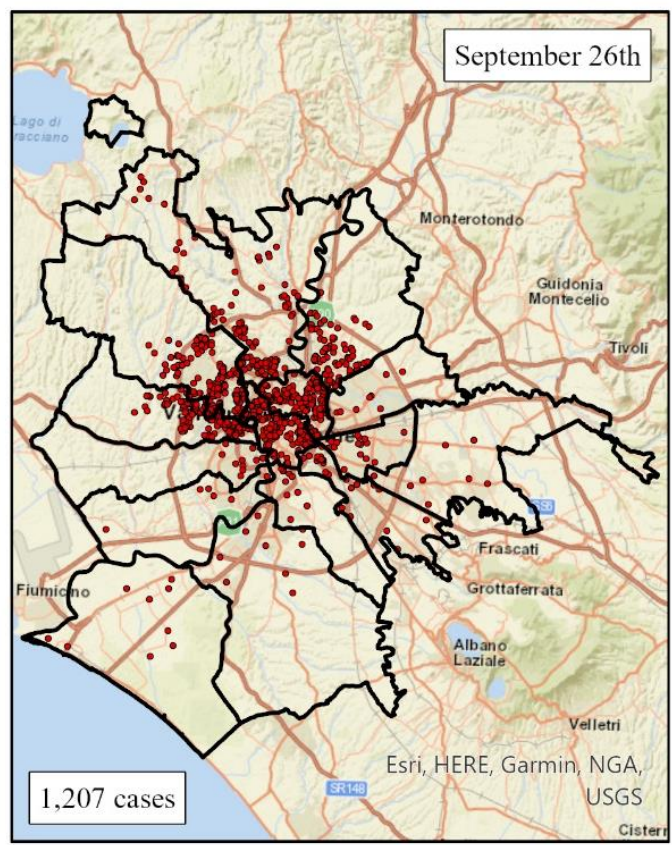

Fig. 4. Four screenshots extracted by the simulator regarding the number of cases of COVID-19 in Rome, for the period June 12th - September 26th, on the basis of the data provided by the UOC Hygiene and Public Health Service - Local Health Unit Rome 1, over a digital street map and with sub-municipal areas of Rome. 

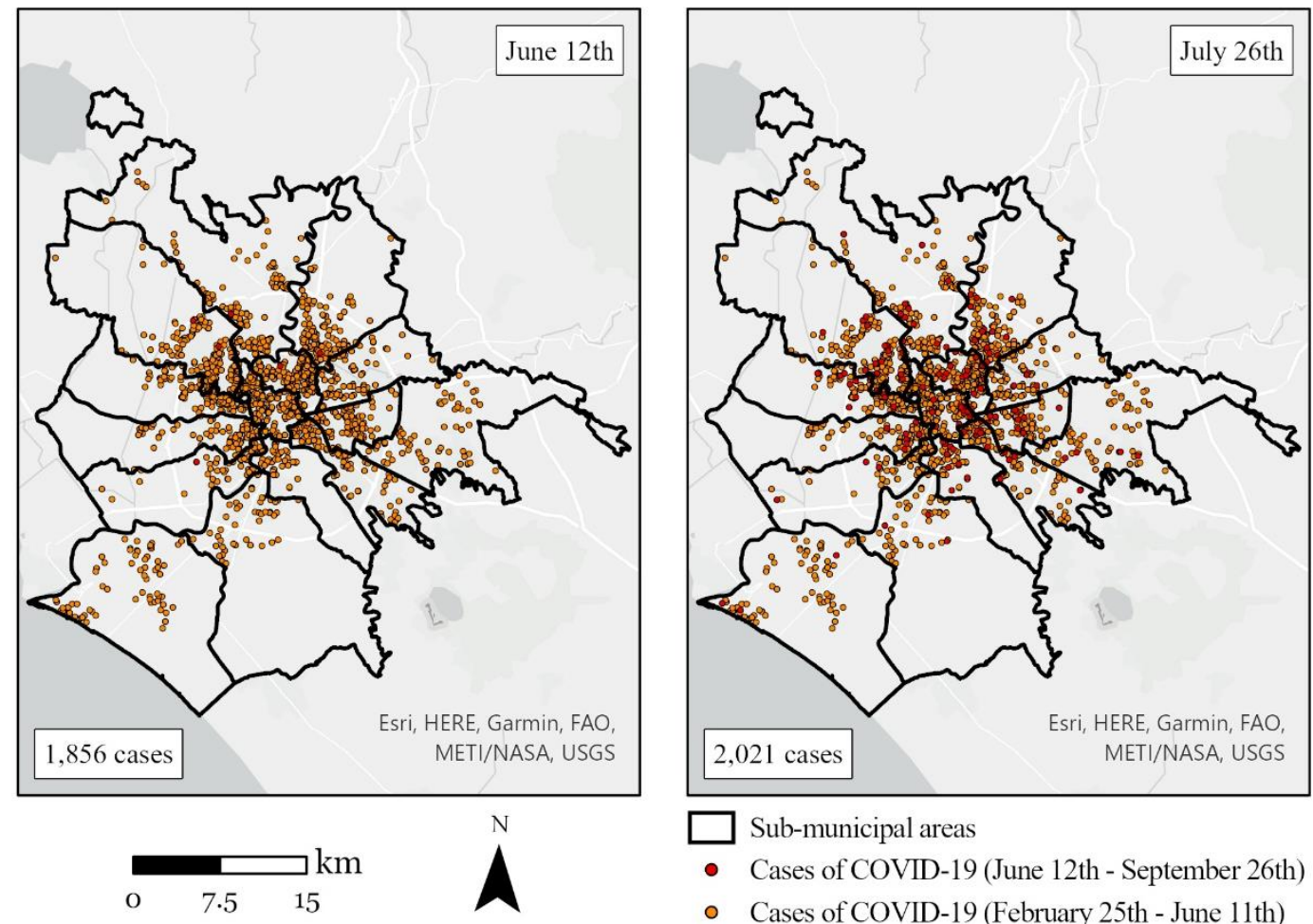

Sub-municipal areas

- Cases of COVID-19 (June 12th - September 26th)

- Cases of COVID-19 (February 25th - June 11th)
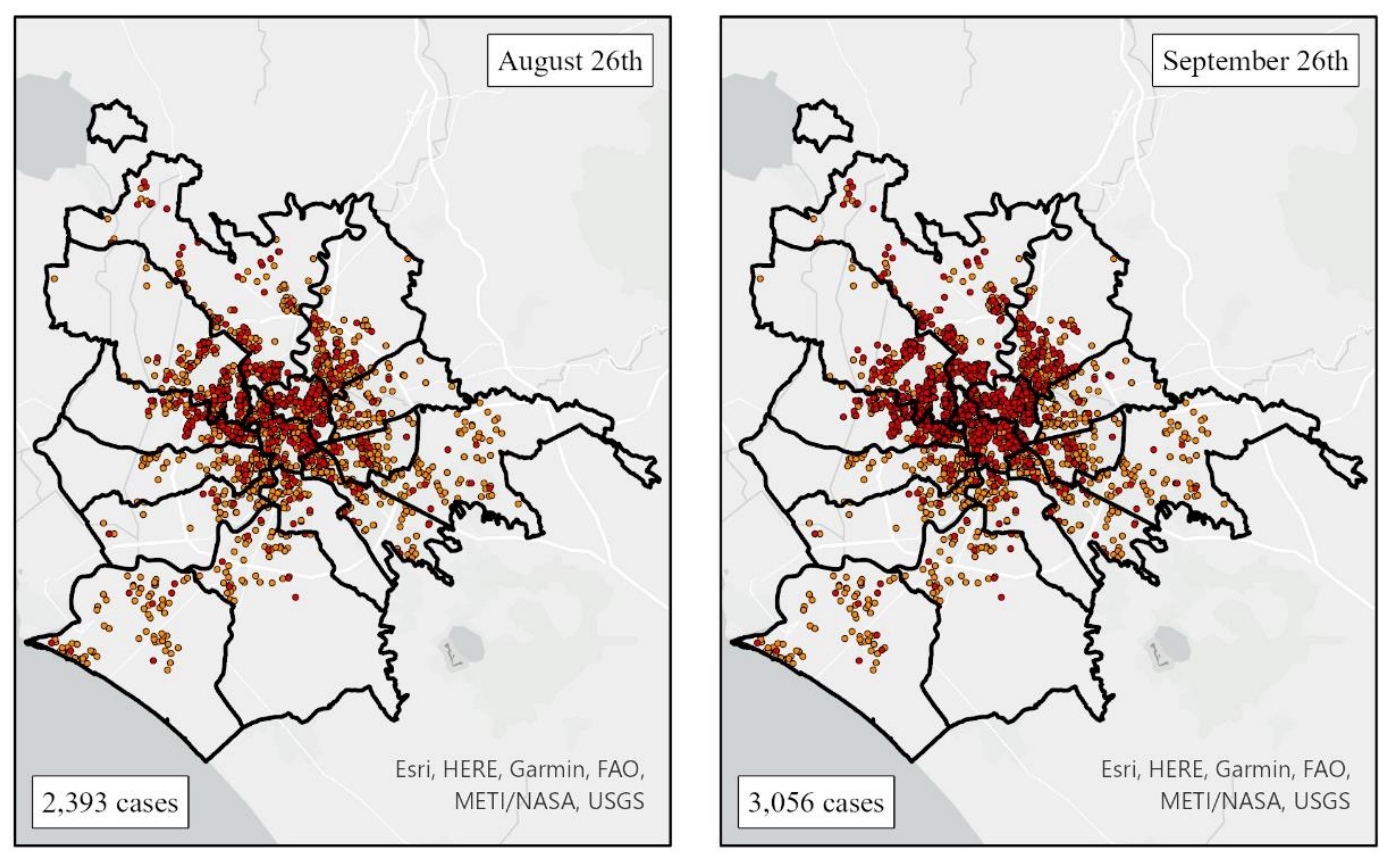

Fig. 5a. Four screenshots extracted by the simulator regarding the total number of cases of COVID-19 in Rome, for the period February 25th - September 26th, on the basis of the data provided by the UOC Hygiene and Public Health Service - Local Health Unit Rome 1, over a Light Gray Canvas as background template and with sub-municipal areas of Rome. The first screenshot, dated June 12th, is due to the sum of the cases recorded from February 25th to June 11th plus the cases recorded on June 12th. 

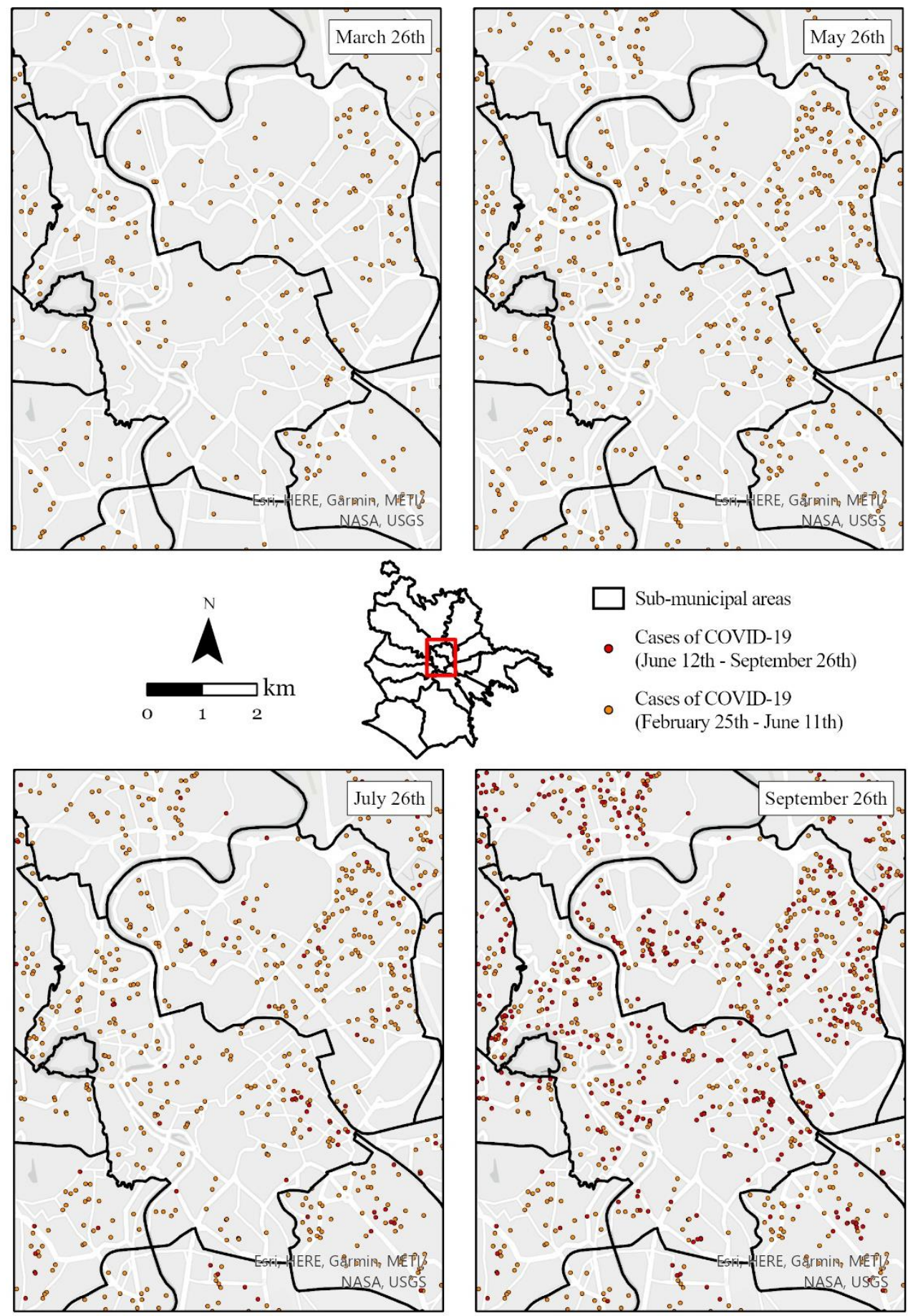

Fig. 5b. Four screenshots extracted by the simulator regarding the total number of cases of COVID-19 in a zone of the Rome centre, for the period March 26th - September 26th, on the basis of the data provided by the UOC Hygiene and Public Health Service - Local Health Unit Rome 1, over a Light Gray Canvas as background template and with sub-municipal areas of Rome. 
In order to provide an exemplifying contribution at detailed geographical scale, with Fig. $\mathbf{5 b}$ we have focussed the attention on a zone of Rome centre, extracting by the simulator four screenshots with bimonthly cadence. It is possible to appreciate the analytical capacity to make in-depth, in terms of applied and interdisciplinary research, intervention planning, targeted closures, interventions weighed on the dynamics in place and distributive patterns. Practically, it is possible to identify - also changing the background template - the streets and buildings where the number of cases is high and tend to rapidly increase, and at the same time it is possible to distinguish clusters and axes which should be immediately kept under particular observation as potential widespread and recharge basins.

\section{CONCLUSIONS}

The here presented dynamic simulator, which can be consulted in video mode on the basis of a specific timeline and according to different time intervals (also every 24 or 48 hours), shows the continuous thickening of points and make it possible to identify, on the basis of the data provided by the UOC Hygiene and Public Health Service - Local Health Unit Rome 1, clusters, patterns and trends, underlining the importance of having a similar application in the surveillance and analysis of the disease.

This simulator is also predisposed and configured to represent only the new cases and remove from the general count those recovered and/or the deceased, through a system of switching and turning off. A similar function makes it possible to geovisualise only the new criticalities, progressively focussing the attention on the different thickening areas with the time pass and evidencing key elements concerning the different weeks, months and waves.

Moreover, the simulator is predisposed to obtain aggregate data i.e. for census sections (CS), census areas (CEA) and sub-municipal areas (SCA), also with the addition of graphs to represent the data subdivided by sex and classes of age. Therefore, it is possible to elaborate choropleth maps that become very useful above all in the case of many data which tend to overlap for the number of cases and the proximity of the addresses. Similar choropleth maps, which can derive from geocoding processes and dot maps, return explanatory aggregate digital representations and would be able to illustrate indexes i.e. built as a ratio between the number of cases and number of residents (also according to the age of the population), number of cases and number of beds in intensive care, number of deaths due to COVID-19 and total deaths etc., above all if a harmonic and interactive system of data collection was set up and in relation to demographic and social-healthcare data of official statistics.

Among the various other perspectives that are opened for future research (reducing the geographical scale) and that can draw added value from models and simulators like the ones here presented are also those of correlating data on positives and mortality-lethality with data on environmental aspects inferable by remote sensing and Earth observation, such as temperatures and heat waves, humidity and precipitation, pressure and winds speed, pollution and the presence of fine dust (which can be loaded as superimposable layers).

In fact, disease digital mapping and GIS methods for efficacious exploration, modelling and visualisation can be used not only to detect, identify and show spatial and temporal distribution, clusters and hotspots of COVID-19, but also to explore and analyse relationships of COVID-19 with other factors, revealing a wide set of the most up-to-date applied approaches in the fields of health and medical geography and spatial epidemiology (Fatima et al., 2021, 11-12).

We agree that detailed spatial-temporal mapping and analysis provide a significant picture regarding where the confirmed cases of COVID-19 tend to be concentrated and can also help to evaluate and verify possible correlations. From the quantitative and qualitative points of view it is possible to differentiate in dynamic digital elaborations and video modes the areas with the highest concentration of cases from the zones with low concentration and the territorial contexts that are in the transition range; so this applicative and scientific approach proves to be relevant in supporting social, health and sanitary actions and for working in the perspectives of real-time surveillance and contagion prevention (da Silva et al., 2021, 15). At the same time, it can be useful to increase and 
optimise the location of integrative health services and structures in specific places and to improve health care delivery with ad hoc measures (Dumitrache et al., 2020, 1). Therefore, painstakingly studying the spatial-temporal dynamics of COVID-19 is a key action to its mitigation, and GIS elaboration, disease mapping and interdisciplinary data analysis are becoming recurrent topics, also in order to inspire new beneficial researches, models and reflections (Franch-Pardo et al., 2020, 9).

We hope that the dynamic space-time diffusion simulator in a GIS environment here presented to tackle the COVID-19 emergency and the relative foundations of application methodology can provide inputs also in the direction of experimenting innovative and harmonised geotechnological solutions to be ready, with quick and effective reaction, in the case of ill-fated future need.

\section{ACKNOWLEDGEMENTS}

This paper has been written together by the authors, but particularly: C. Pesaresi has written sections 2, 3.1, 3.5, 4.1, 4.2.1 and 5 and he is co-author of section 4.2.3; Davide Pavia has written sections 3.3, 3.4, 4.2.2 and he is coauthor of section 4.2.3; Corrado De Vito has written section 1; Andrea Barbara, Vito Cerabona and Enrico Di Rosa have written section 3.2 and made the data available.

\section{R E F E R E N C E S}

Aschwanden, C. (2021) Five reasons why COVID herd immunity is probably impossible. Nature, 591, 520-522. Atek, S., Pesaresi, C., Eugeni, M., Gaudenzi, P., De Vito, C., Cardinale, V., Mecella, M., Maraschini, A., Pistillo, P. \& Vora, A. (2021) An Earth Observation Cognitive System in Response to Sars-Covid-19 Emergency. 72nd International Astronautical Congress 2021, Abstract, Paper ID: 64144 oral.

Avand, M., Moradi, H. \& Ramazanzadeh lasboyee, M. (2021) Using machine learning models, remote sensing, and GIS to investigate the effects of changing climates and land uses on flood probability. Journal of Hydrology, 595, 125663.

Cosgriff, C.V., Ebner, D.K. \& Celi, L.A. (2020) Data sharing in the era of COVID-19. Lancet Digit Health, 2, $5, \mathrm{e} 224$.

Cristea, C. \& Jocea, A.F. (2016) GIS Application for Wind Energy. Energy Procedia, 85, 132-140.

Dangermond, J., De Vito, C. \& Pesaresi, C. (2020) "Using GIS in the Time of the COVID-19 Crisis, casting a glance at the future. A joint discussion". J-READING (Journal of Research and Didactics in Geography), $1,9,195-205$.

Dardanelli, G., Marretta, R., Santamaria, A.S., Streva, A., Lo Brutto, M. \& Maltese, A. (2017) Analysis of Technical Criticalities for GIS Modelling an Urban Noise Map. Geographia Technica, 12, 2, 41-61.

da Silva, C.C. et al. (2021) Covid-19 Dynamic Monitoring and Real-Time Spatio-Temporal Forecasting. Frontiers in Public Health, 9, 1-17.

de Kadt, J., Götz, G., Hamann, C., Maree, G. \& Parker, A. (2020) Mapping vulnerability to COVID-19 in Gauteng. GCRO Map of the Month, Gauteng City-Region Observatory. Available at https://gcro.ac.za/outputs/map-of-the-month/detail/mapping-vulnerability-to-covid-19/.

Desjardins, M.R., Hohl, A. \& Delmelle, E.M. (2020) Rapid surveillance of COVID-19 in the United States using a prospective space-time scan statistic: Detecting and evaluating emerging clusters. Applied Geography, $118,1-7$.

Dumitrache, L., Nae, M., Simion, G. \& Talos, A.-M. (2020) Modelling Potential Geographical Access of the Population to Public Hospitals and Quality Health Care in Romania. International Journal of Environmental Research and Public Health, 17, 1-18.

Fatima, M., O'Keefe, K.J., Wei, W., Arshad, S. \& Gruebner, O. (2021) Geospatial Analysis of COVID-19: A Scoping Review. International Journal of Environmental Research and Public Health, 18, 1-14.

Franch-Pardo, I., Napoletano, B.M., Rosete-Verges, F. \& Billa, L. (2020) Spatial analysis and GIS in the study of COVID-19. A review. Science of the Total Environment, 739, 1-10. 
Horton, R. (2021) Offline: The lessons of smallpox eradication for COVID-19. Lancet, 2021, 3961951.

Kamel Boulos, M.N. \& Geraghty, E.M. (2020) Geographical tracking and mapping of coronavirus disease COVID-19/severe acute respiratory syndrome coronavirus 2 (SARS-CoV-2) epidemic and associated events around the world: how 21st century GIS technologies are supporting the global fight against outbreaks and epidemics. International Journal of Health Geographics, 19, 8.

Kupferschmidt, K. (2020) Europe is locking down again-but its strategy is unclear. Science, 370, 644-645.

Lloyd-Sherlock, P., Sempe, L., McKee, M. \& Guntupalli, A. (2021) Problems of Data Availability and Quality for COVID-19 and Older People in Low- and Middle-Income Countries. Gerontologist, 61, 2, $141-144$.

Looi, M.K. (2020) COVID-19: Is a second wave hitting Europe? BMJ, 371, m4113.

McGill COVID19 Vaccine Tracker Team (2021) COVID19 Vaccine Tracker. Available at https://covid19.trackvaccines.org/.

Ministero della Salute (2021). Report Vaccini Anti COVID-19. Available at www.governo.it/it/cscovid19/reportvaccini/.

Nistor, M.-M., Rahardjo, H., Satyanaga, A., Leong, E., Hao, K.Z., Sham, A.W.L. \& Wu, H. (2019) GIS-Based Approach to Identify the Suitable Locations for Soil Sampling in Singapore. Geographia Technica, 14, 1, $103-117$.

Pesaresi, C. (2020) A geographical and crosscutting look at the COVID-19 pandemic in an international framework. Introduction. J-READING - Journal of Research and Didactics in Geography, 2, 9, 13-19.

Pesaresi, C., Migliara, G., Pavia, D. \& De Vito, C. (2020a) Emergency Department Overcrowding: A Retrospective Spatial Analysis and the Geocoding of Accesses. A Pilot Study in Rome. ISPRS International Journal of Geo-Information, 9, 579, 1-26.

Pesaresi, C. \& Pavia, D. (2021) Radio Base Stations and Electromagnetic Fields: GIS Applications and Models for Identifying Possible Risk Factors and Areas Exposed. Some Exemplifications in Rome. ISPRS International Journal of Geo-Information, 10, 3, 1-21.

Pesaresi, C., Pavia, D. \& De Vito, C. (2020b) Three geotechnological proposals to tackle health emergencies and the monitoring of infectious diseases. Inputs from the COVID-19 pandemic for future preparedness. Bollettino della Associazione Italiana di Cartografia, 170.

Riffe, T., Acosta, E. \& COVerAGE-DB project team (2020) COVerAGE-DB: A database of age-structured COVID-19 cases and deaths. Preprint available at https://www.medrxiv.org/content/10.1101/2020.09.18.20197228v2.full.pdf, 1-13.

Smith, C.D. \& Mennis, J. (2020) Incorporating Geographic Information Science and Technology in Response to the COVID-19 Pandemic. Preventing Chronic Disease, 17, E58, 1-7.

Tse, E.G., Klug, D.M. \& Todd, M.H. (2020) Open science approaches to COVID-19. F1000Research, 9, 1043, $1-6$.

Valjarević, A., Milić, M., Valjarević, D., Stanojević-Ristić, Z., Petrović, L., Milanović, M., Filipović, D., Ristanović, B., Biljana, B. \& Lukić, T. (2020) Modelling and mapping of the COVID-19 trajectory and pandemic paths at global scale: A geographer's perspective. Open Geosciences, 12, 1603-1616.

Wolkewitz, M. \& Puljak, L. (2020) Methodological challenges of analysing COVID-19 data during the pandemic. BMC Medical Research Methodology, 20, 81, 1-4.

Xiong, Y., Wang, Y., Chen, F. \& Zhu, M. (2020) Spatial Statistics and Influencing Factors of the COVID-19 Epidemic at Both Prefecture and County Levels in Hubei Province, China. International Journal of Environmental Research and Public Health, 17, 1-26.

Xu, M., Cao, C., Zhang, X., Lin, H., Yao, Z., Zhong, S., Huang, Z. \& Duerler, R.S. (2021) Fine-Scale SpaceTime Cluster Detection of COVID-19 in Mainland China Using Retrospective Analysis. International Journal of Environmental Research and Public Health, 30, 18, 3583. 